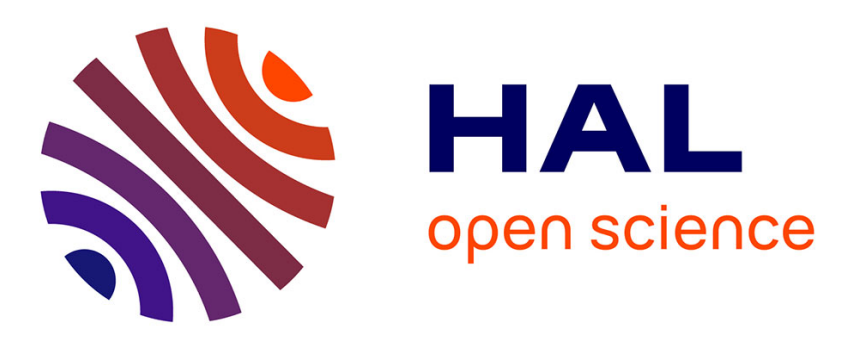

\title{
Cyclic Autocorrelation based Spectrum Sensing: Theoretical Derivation Framework
}

Vincent Gouldieff, Amor Nafkha, Nicolas Grollier, Jacques Palicot, Steredenn

Daumont

\section{- To cite this version:}

Vincent Gouldieff, Amor Nafkha, Nicolas Grollier, Jacques Palicot, Steredenn Daumont. Cyclic Autocorrelation based Spectrum Sensing: Theoretical Derivation Framework. 2018 25th International Conference on Telecommunications (ICT), Jun 2018, Saint-Malo, France. 10.1109/ICT.2018.8464887 . hal-02280735

\section{HAL Id: hal-02280735}

\section{https://imt-atlantique.hal.science/hal-02280735}

Submitted on 6 Sep 2019

HAL is a multi-disciplinary open access archive for the deposit and dissemination of scientific research documents, whether they are published or not. The documents may come from teaching and research institutions in France or abroad, or from public or private research centers.
L'archive ouverte pluridisciplinaire $\mathbf{H A L}$, est destinée au dépôt et à la diffusion de documents scientifiques de niveau recherche, publiés ou non, émanant des établissements d'enseignement et de recherche français ou étrangers, des laboratoires publics ou privés. 


\title{
Cyclic Autocorrelation based Spectrum Sensing: Theoretical Derivation Framework
}

\author{
Vincent Gouldieff ${ }^{1,2}$, Amor Nafkha $^{1}$, Nicolas Grollier $^{3}$, Jacques Palicot ${ }^{1}$, Steredenn Daumont ${ }^{2}$ \\ ${ }^{1}$ CentraleSupelec/IETR, Avenue de la Boulaie - CS 47601, 35576 Cesson-Sevigne Cedex, France \\ ${ }^{2}$ Zodiac Data Systems, 2 Rue de Caen, 14740 Bretteville-l'Orgueilleuse, France \\ ${ }^{3}$ Lab-STICC, CNRS, IMT Atlantique, 29238 Brest, France \\ Email: firstname.lastname@\{centralesupelec.fr, zodiacaerospace.com, imt-atlantique.fr\}
}

\begin{abstract}
In this article, we propose a theoretical framework to derive the stochastic behavior of the cyclic autocorrelation power (CAP). This function is especially used in cyclostationaritybased spectrum sensing for its robustness to noise uncertainty and its low computational cost. We first express the theoretical probability density function (PDF) of the cyclic autocorrelation power - which proves to follow a central scaled (respectively noncentral) chi-square distribution if the received samples consist of additive Gaussian noise (respectively noise plus a cyclostationary signal). In order to verify the accuracy of the proposed theoretical derivation, simulation results are then provided in terms of detection and false alarm probabilities.
\end{abstract}

Index Terms-Cyclic autocorrelation function, cyclostationarity, spectrum sensing, theoretical distributions.

\section{INTRODUCTION}

Cyclostationarity-based signal processing is a major advance in blind detection and estimation of communication signals. Consequently, it is of real interest for cognitive radio systems and it is widely used for spectrum sensing [1], timing estimation [2], source localization [3], automatic modulation classification (AMC) [4], and so on. Its performances mainly rely on the way the signal-of-interest - which exhibits some cyclostationary properties - shows particular features even if it is highly corrupted by some stationary noise - which would asymptotically (i.e. when the observation length goes to infinity) vanish with appropriately chosen transforms.

In the literature, several functions have been proposed to bring the cyclostationary property out. For instance, cyclicmoments (CM) and cyclic-cumulants (CC) are commonly used in AMC [5]. Meanwhile, the so-called $M^{t h}$-Power nonlinear Transformation (MPT) [2][6] is useful for both symbol timing detection and AMC. Last but not least, cyclic autocorrelation function (CAF) [7], spectral correlation density (SCD) [8] and spectrum coherence (SC) [9] have shown numerous benefits in the field of spectrum sensing.

In order to design a detection (or estimation) algorithm and to predict its performance, one may be interested in deriving its theoretical behavior. This derivation necessarily arises from the study of the probabilistic distribution of the chosen transformation, given a set of assumptions and parameters.

In cyclostationarity-based spectrum sensing, a set of features is generally extracted from a two dimensional cyclic function (CAF, CAP, SCD, SC...). A detector (statistical test, threshold, specific property...) finally makes a decision about the presence or absence of a certain type of signal in the collected samples. For the aforementioned purpose, obtaining the theoretical performances in terms of probability of detection $\left(P_{d}\right)$ and probability of false alarm $\left(P_{f a}\right)$ is usually needed to verify that a given detector would match some performance requirements. For instance, the IEEE 802.22 norm requires a $P_{d}$ of 0.9 for a $P_{f a}$ of 0.1 at $-20.8 \mathrm{~dB}$ SNR [10].

In the litterature, several theoretical studies in this direction have been carried out. For instance, we may refer to the pioneering articles by Gardner and Spooner [8], and by Dandawate and Giannakis [11]. However, these studies are more focused on the asymptotic behavior of the cyclostationaritybased detectors than on there probabilistic behavior, i.e. when a short observation length is considered. More recently, and mainly based on the mathematical results provided in the book by S. Mac Kay [12], some novel methods have been proposed to derive approximate distributions in the context of spectral correlation density-based detectors [13] or in the case of classical first or second-order cyclic detectors [14].

To the best of our knowledge, no attempt to derive the theoretical distributions of the cyclic autocorrelation power (CAP) was made. However, such a study is of real interest for two reasons. First, simulation results show that the CAP has good performance and robustness when used in spectrum sensing. Secondly, the CAP is particularly cost-effective, which makes it a great tool for embedded cognitive receiver applications.

In this paper, we propose a framework to derive the theoretical distributions of the CAP. Then, we apply these results in the context of spectrum sensing. Our work mainly relies on the study by J. E. Mazo [2] - carried out in the case of the MPT for timing estimation purpose - and continued by J. Reichert in [15] - in the context of AMC. As in [14], our study follows a two steps approach: we first derive the asymptotic behavior of the CAP. It is then used to derive the distributions of the CAP in the finite-length observation context.

The paper is organized as follows: In Section II the problem under study is formulated, while in Section III the asymptotic study of the CAP is conducted. In Section IV the theoretical development of the distributions is carried out. Finally, in Section V, the proposed theoretical results are confronted to simulations in the context of spectrum sensing. Section VI concludes the paper. 


\section{CAP-BASED SPECTRUM SENSING: SYSTEM MODEL}

We first list the assumptions made throughout the paper. We also briefly present the properties exhibited by the CAP for a better understanding of cyclostationarity-based detection.

\section{A. Spectrum sensing problem formulation}

The spectrum sensing problem consists in determining if a primary user is present or absent in a given frequency band $B$ during a given time interval. This problem is solved by collecting a vector of $N$ data samples $\mathbf{y}=[y(1), \ldots, y(N)]$ and by considering the following two hypotheses problem: namely, $\mathcal{H}_{0}$ - respectively $\mathcal{H}_{1}$ - corresponds to the absence respectively presence - of a primary user.

We consider the situation where the primary user is a pulse-shaped digital signal transmitted over an additive white gaussian noise (AWGN) channel. Then, the entailed binary problem is defined such that, under $\mathcal{H}_{0}, \mathbf{y}$ is only composed of noise samples, while under $\mathcal{H}_{1}, \mathbf{y}$ is composed of noise samples plus the samples of the signal from the primary user.

Hence, the detection problem is formulated as: make a decision between $\mathcal{H}_{0}$ and $\mathcal{H}_{1}$ such that for all $n$ in $\{1, \ldots, N\}$,

$$
\left\{\begin{array}{l}
\mathcal{H}_{0}: y(n)=\omega(n) \\
\mathcal{H}_{1}: y(n)=x(n)+\omega(n)
\end{array}\right.
$$

where $x(n)=\sum_{k} s(k) \cdot h(n-\rho k)$. The series $\mathbf{s}$ consists of indepedent and identically distributed symbols carried by zero mean and unit variance constellation $C$. Vector $\mathbf{h}$ is the equivalent channel (i.e. the convolution of the pulse-shaping function and the physical channel) and $\rho$ is the oversampling factor. Finally, $\omega(n)$ denotes the additive channel noise.

In this paper, we assume that $\mathbf{h}$ is a centered rectangular pulse-shaping function with unit normalized length, while $\omega(n)$ is modelled as a complex zero-mean white Gaussian noise with variance $\sigma_{\omega}^{2}$, i.e. $\omega(n) \sim \mathcal{N}_{C}\left(0, \sigma_{\omega}^{2}\right)$. The extension of the proposed framework to other types of channels or signals should be straightforward.

\section{B. Basic considerations related to the CAP}

Let consider the equivalent continuous-time signal $x(t)$ as

$$
x(t)=\sum_{k} s(k) \cdot h\left(t-k T_{s}\right),
$$

where $T_{s}$ stands for the symbol duration.

Under weak assumptions on equivalent channel $h$, the random process $x(t)$ proves to be wide-sense second-order (almost)-cyclostationary [16]. Hence, the cyclic autocorrelation function (CAF) of signal $x$ corresponds to the Fourier coefficients of the classical autocorrelation function $R_{x x}\left(\tau_{0}\right)$. Then, the CAF may be written as in [7] as

$$
R_{x x}^{\alpha}\left(\tau_{0}\right)=\lim _{T \rightarrow \infty} \frac{1}{T} \int_{-T / 2}^{T / 2} x\left(t+\frac{\tau_{0}}{2}\right) x^{*}\left(t-\frac{\tau_{0}}{2}\right) e^{-j 2 \pi \alpha t} d t .
$$

Based on the CAF, the detection process may rely on the spectral lines that appear in the cyclic autocorrelation power (CAP) - denoted as $\mathcal{S}_{x x}^{\alpha}\left(\tau_{0}\right) \triangleq\left|R_{x x}^{\alpha}\left(\tau_{0}\right)\right|^{2}$ - at each integer multiple of the cyclic frequency (i.e. when $\alpha \in T_{s}^{-1} \mathbb{Z}$ ).
As a consequence, our goal is to derive the distribution of the cyclic autocorrelation power $\mathcal{S}_{y y}^{\alpha}\left(\tau_{0}\right)$ for $\left\{\alpha, \tau_{0}\right\} \in \mathbb{R} \times \mathbb{R}^{*}$, under both assumption $\mathcal{H}_{0}$ and $\mathcal{H}_{1}$. For this purpose, we note that $\mathcal{S}_{y y}^{\alpha}\left(\tau_{0}\right)$ can also be written as the Fourier transform of the autocorrelation of function

$$
y y\left(t, \tau_{0}\right) \triangleq y\left(t+\frac{\tau_{0}}{2}\right) y^{*}\left(t-\frac{\tau_{0}}{2}\right)
$$

as

$$
\mathcal{S}_{y y}^{\alpha}\left(\tau_{0}\right)=\int_{-\infty}^{+\infty} r_{y y\left(t, \tau_{0}\right)}(\tau) e^{-i 2 \pi \alpha \tau} d \tau
$$

where we denoted

$$
r_{y y\left(t, \tau_{0}\right)}(\tau)=\frac{1}{T_{s}} \int_{0}^{T_{s}} \mathbb{E}\left[y y\left(t+\frac{\tau}{2}, \tau_{0}\right) y y^{*}\left(t-\frac{\tau}{2}, \tau_{0}\right)\right] d t .
$$

\section{CONTINUOUS-TIME OBSERVATION MODEL}

We first develop the theoretical asymptotic expression of $\mathcal{S}_{y y}^{\alpha}\left(\tau_{0}\right)$ - i.e. when $y$ is seen as a continuous-time infinitelength random process. In this context, we first consider that $y$ is the continuous signal from which the $N$ samples described in Section II. A. are collected. Thus, for all $t, y(t)$ is such that

$$
\left\{\begin{array}{l}
\mathcal{H}_{0}: y(t)=\omega(t) \\
\mathcal{H}_{1}: y(t)=\sum_{k} s(k) \cdot h\left(t-k T_{s}\right)+\omega(t)
\end{array}\right.
$$

where $T_{s}$ is the symbol duration and $h(t)$ is the unit-power rectangular function between $-T_{s} / 2$ and $T_{s} / 2$.

\section{A. Cyclic autocorrelation power under $\mathcal{H}_{0}$}

Under $\mathcal{H}_{0}$ and in the presence of white Gaussian noise, it is trivial to show that for all $\left\{\alpha, \tau_{0}\right\} \in \mathbb{R} \times \mathbb{R}^{*}$

$$
\mathcal{S}_{y y \mid \mathcal{H}_{0}}^{\alpha}\left(\tau_{0}\right)=\sigma_{\omega}^{4}
$$

where we recall that $\sigma_{\omega}^{2}$ stands for the variance of $\omega$.

\section{B. Cyclic autocorrelation power under $\mathcal{H}_{1}$}

We first develop the product $y y\left(t, \tau_{0}\right)$ as a sum of crossterms as in [13]. Since $x$ and $\omega$ are mutually independent, we may get for all $\left\{\alpha, \tau_{0}\right\} \in \mathbb{R} \times \mathbb{R}^{*}$,

$$
\mathcal{S}_{y y \mid \mathcal{H}_{1}}^{\alpha}\left(\tau_{0}\right)=\mathcal{S}_{x x}^{\alpha}\left(\tau_{0}\right)+2 \mathcal{S}_{x \omega}^{\alpha}\left(\tau_{0}\right)+\mathcal{S}_{\omega \omega}^{\alpha}\left(\tau_{0}\right)
$$

Due to the independence between $x(t)$ and $\omega(t)$, we also have $\mathcal{S}_{x \omega}^{\alpha}\left(\tau_{0}\right)=\sigma_{\omega}^{2}$. Moreover, it can be shown that $\mathcal{S}_{\omega \omega}^{\alpha}\left(\tau_{0}\right)=\sigma_{\omega}^{4}$, yields the following expression for (7):

$$
\mathcal{S}_{y y \mid \mathcal{H}_{1}}^{\alpha}\left(\tau_{0}\right)=\mathcal{S}_{x x}^{\alpha}\left(\tau_{0}\right)+2 \sigma_{\omega}^{2}+\sigma_{\omega}^{4} .
$$

Hence, the only unknown term in (7) is $\mathcal{S}_{x x}^{\alpha}\left(\tau_{0}\right)$. Following the work carried out by Mazo [2], we may get the theoretical expression of this term from (5) by splitting $x$ into its random part (involved by symbols $s$ ) and into its deterministic part (involved by invariant channel $h$ ). 
1) Derivation of the random part: Under the i.i.d. assumption for symbol stream $s$ carried by constellation $C$, we may show that for all $\{a, b, c, d\} \in \mathbb{Z}^{4}$ we have

$$
\begin{aligned}
\mathbb{E}\left[s(a) s^{*}(b) s^{*}(c) s(d)\right]= & \delta_{a b} \delta_{c d}+\delta_{a c} \delta_{b d}+\mu_{2}^{2} \cdot \delta_{a d} \delta_{b c} \\
& -\left(2+\mu_{2}^{2}-\mu_{4}^{*}\right) \cdot \delta_{a b} \delta_{a c} \delta_{a d}
\end{aligned}
$$

where $\delta_{a b}$ is the Kronecker delta function,

$$
\delta_{a b}= \begin{cases}1, & \text { if } a=b \\ 0, & \text { else }\end{cases}
$$

$\mu_{2}$ is the second-order moment of $C$ and $\mu_{4}^{*}$ is its fourth order two-conjugated moment. You may refer to Table I for the values of the coefficients classically considered in (9).

TABLE I

CONSIDERED COEFFICIENTS IN (9)

\begin{tabular}{|c|c|c|c|c|c|}
\hline Constellation $C \rightarrow$ & BPSK & QPSK & 8PSK & 16QAM & 4PAM \\
\hline \hline$\mu_{2}^{2}$ & 1 & 0 & 0 & 0 & 1 \\
\hline$\mu_{4}^{*}$ & 1 & 1 & 1 & $\frac{33}{25}$ & $\frac{41}{25}$ \\
\hline $2+\mu_{2}^{2}-\mu_{4}^{*}$ & 2 & 1 & 1 & $\frac{17}{25}$ & $\frac{34}{25}$ \\
\hline
\end{tabular}

2) Derivation of the deterministic part: Related to the four terms in (9), we now derive the four cyclic autocorrelation terms that take part in the following equation

$$
\begin{gathered}
\mathcal{S}_{y y \mid \mathcal{H}_{1}}^{\alpha}\left(\tau_{0}\right)=\mathcal{S}_{a b, c d}^{\alpha}\left(\tau_{0}\right)+\mathcal{S}_{a c, b d}^{\alpha}\left(\tau_{0}\right)+\mu_{2}^{2} \cdot \mathcal{S}_{a d, b c}^{\alpha}\left(\tau_{0}\right) \\
-\left(2+\mu_{2}^{2}-\mu_{4}^{*}\right) \cdot \mathcal{S}_{a b, a c, a d}^{\alpha}\left(\tau_{0}\right)+2 \sigma_{\omega}^{2}+\sigma_{\omega}^{4}
\end{gathered}
$$

where $\{a, b, c, d\} \in \mathbb{Z}^{4}$ as in (9) and where the literal expression of each CAP may be derived as detailed in Appendix.

For instance, if $h$ is a centered unit-power rectangular pulseshape, we may get for the first term

$$
\begin{aligned}
\mathcal{S}_{a b, c d}^{\alpha}\left(\tau_{0}\right)= & \left(\frac{T_{s}-\tau_{0}}{T_{s}}\right)^{2} . \\
& \sum_{m \in f_{s} \mathbb{Z}} \operatorname{sinc}^{2}\left[\left(T_{s}-\tau_{0}\right) m\right] \delta(\alpha-m)
\end{aligned}
$$

where $\delta(\cdot)$ stands for the Dirac delta function and $f_{s}=T_{s}^{-1}$.

We may note that the presence of spectral lines in the cyclic autocorrelation power may be partly explained by (12) as suggested by the presence of a sum of Dirac delta functions, each of them shifted by a multiple of the cyclic frequency.

The derivation of the three other cyclic autocorrelation terms follows the same principle and should not be an issue. We especially get with a rectangular pulse:

$$
\mathcal{S}_{a c, b d}^{\alpha}\left(\tau_{0}\right)=\left(\frac{\tau_{0}}{T_{s}}\right)^{2} \operatorname{sinc}^{2}\left(\frac{\tau_{0}}{T_{s}} \cdot \alpha\right)
$$

and

$$
\begin{aligned}
\mathcal{S}_{a d, b c}^{\alpha}\left(\tau_{0}\right)= & \mathcal{S}_{a b, a c, a d}^{\alpha}\left(\tau_{0}\right)= \\
& \left(\frac{T_{s}-\tau_{0}}{T_{s}}\right)^{2} \operatorname{sinc}^{2}\left(\frac{T_{s}-\tau_{0}}{T_{s}} \cdot \alpha\right) .
\end{aligned}
$$

\section{DisCRETE FINITE-LENGTH OBSERVATION MODEL}

In the spectrum sensing context, the value of $N$ is relatively small so that the infinite-length model does no longer hold. Then, based on the development proposed in Section III, we derive the theoretical distribution of $\mathcal{S}_{y y}^{\alpha}\left(n_{0}\right)$ under both $\mathcal{H}_{0}$ and $\mathcal{H}_{1}$. The discrete-time CAP of $y$ may be defined as

$$
\mathcal{S}_{y y}^{\alpha}\left(n_{0}\right) \triangleq N^{-1} \cdot\left|\mathcal{F}_{n}\left[y(n) \cdot y^{*}\left(n-n_{0}\right)\right](\alpha)\right|^{2}
$$

where $\mathcal{F}_{n}$ stands for the discrete-time Fourier transform (DTFT) with respect to $n$ and $n_{0} \in \llbracket 1 ;\lfloor\rho\rfloor \rrbracket$ to the delay. We recall that $\rho$ is the oversampling factor and $\lfloor\cdot\rfloor$ stands for the floor function. In all what follows, we denote by $p[\lambda \mid \mathcal{H}](x)$ the PDF of random variable $\lambda$ under hypothesis $\mathcal{H}$.

\section{A. Distribution under $\mathcal{H}_{0}$}

Based on the derivation in [12], it is easy to show that for all normalized $\alpha \in[-\rho / 2 ; \rho / 2], \mathcal{S}_{y y}^{\alpha}\left(n_{0}\right)$ follows a scaled Chi-square distribution with two degrees of freedom.

It follows from (6) that we have, whatever $\left\{\alpha, n_{0}\right\}$

$$
p\left[\mathcal{S}_{y y}^{\alpha}\left(n_{0}\right) \mid \mathcal{H}_{0}\right](x)=\frac{1}{\theta_{0}} \cdot e^{-\frac{x}{\theta_{0}}} .
$$

where $\theta_{0}$ is necessarily equal to $\sigma_{\omega}^{4}$.

\section{B. Distributions under $\mathcal{H}_{1}$}

Thereby, two situations have to be dealt with. We especially have to consider whether the value of $\alpha$ is a multiple of the normalized cyclic frequency - i.e. $\alpha \in \mathbb{Z}$ - or not.

We first define the two following power spectral functions $\mathcal{S}_{1}^{\alpha}\left(n_{0}\right)$ and $\mathcal{S}_{2}^{\alpha}\left(n_{0}\right)$ as

$$
\begin{gathered}
\mathcal{S}_{1}^{\alpha}\left(n_{0}\right)=\left(\frac{\rho-n_{0}}{\rho}\right)^{2} \cdot\left(\frac{\operatorname{sinc}\left(\alpha \cdot\left(\rho-n_{0}\right) \cdot \rho^{-1}\right)}{\operatorname{sinc}\left(\alpha \cdot \rho^{-1}\right)}\right)^{2}, \\
\mathcal{S}_{2}^{\alpha}\left(n_{0}\right)=\left(\frac{n_{0}}{\rho}\right)^{2} \cdot\left(\frac{\operatorname{sinc}\left(\alpha \cdot n_{0} \cdot \rho^{-1}\right)}{\operatorname{sinc}\left(\alpha \cdot \rho^{-1}\right)}\right)^{2} .
\end{gathered}
$$

Equations (17) and (18) correspond to the discrete-time finite-length adaptations of the basis of functions obtained in Section III. B. 2), namely in (12), (13) and (14).

We especially remark in (17) and (18) the presence of a denominator involving the sinc function, which is due to the truncation of the signal on $N$ samples compared to the infinitelength model. Since the oversampling factor is considered greater than or equal to 1 , the sampling process has no other impact on (17) and (18) than the presence of the oversampling ratio $\rho$ in the expression of both $\mathcal{S}_{1}^{\alpha}\left(n_{0}\right)$ and $\mathcal{S}_{2}^{\alpha}\left(n_{0}\right)$.

In the first case - i.e. $\alpha \in \mathbb{Z}-\mathcal{S}_{y y \mid \mathcal{H}_{1}}^{\alpha}\left(n_{0}\right)$ proves to follow a scaled version of the non-central Chi-square distribution with two degrees of freedom [12], which expression is

$$
p\left[\mathcal{S}_{y y}^{\alpha}\left(n_{0}\right) \mid \mathcal{H}_{1}\right](x)=\frac{1}{\theta_{1}^{\alpha}\left(n_{0}\right)} e^{-\frac{x+\lambda_{1}^{\alpha}\left(n_{0}\right)}{\theta_{1}^{\alpha}\left(n_{0}\right)}} I_{0}\left(\frac{2 \sqrt{x \lambda_{1}^{\alpha}\left(n_{0}\right)}}{\theta_{1}^{\alpha}\left(n_{0}\right)}\right)
$$

where $\lambda_{1}^{\alpha}\left(n_{0}\right)$ stands for the non-centrality parameter and $\theta_{1}^{\alpha}\left(n_{0}\right)$ for the scaling factor. 
Noting that the expectation of (19) is

$$
\mathbb{E}\left[\mathcal{S}_{y y}^{\alpha}\left(n_{0}\right)\right]=\theta_{1}^{\alpha}\left(n_{0}\right)+\lambda_{1}^{\alpha}\left(n_{0}\right)
$$

and that its variance is

$$
\mathbb{V}\left[\mathcal{S}_{y y}^{\alpha}\left(n_{0}\right)\right]=\theta_{1}^{\alpha}\left(n_{0}\right) \cdot\left(\theta_{1}^{\alpha}\left(n_{0}\right)+2 \lambda_{1}^{\alpha}\left(n_{0}\right)\right),
$$

it is possible to derive the theoretical expressions of $\lambda_{1}^{\alpha}\left(n_{0}\right)$ and $\theta_{1}^{\alpha}\left(n_{0}\right)$ by considering that expectation (20) and variance (21) asymptotically tends to their theoretical limits, yields

$$
\lambda_{1}^{\alpha}\left(n_{0}\right)=N \mathcal{S}_{1}^{\alpha}\left(n_{0}\right)+\rho\left(1-\mu_{4}^{*}\right) \mathcal{S}_{1}^{\alpha}\left(n_{0}\right)-\rho \mu_{2}^{2} \mathcal{S}_{2}^{\alpha}\left(n_{0}\right),
$$

and

$$
\begin{aligned}
\theta_{1}^{\alpha}\left(n_{0}\right)=\rho(1+ & \left.\mu_{2}^{2}\right) \mathcal{S}_{2}^{\alpha}\left(n_{0}\right) \\
& -2 \rho\left(1-\mu_{4}^{*}\right) \mathcal{S}_{1}^{\alpha}\left(n_{0}\right)+\sigma_{\omega}^{4}+2 \sigma_{\omega}^{2} .
\end{aligned}
$$

In the spectral background noise case - i.e. $\alpha \notin \mathbb{Z}$ - $\mathcal{S}_{y y \mid \mathcal{H}_{1}}^{\alpha}\left(n_{0}\right)$ proves to follow a scaled central Chi-square distribution with two degrees of freedom. We may have

$$
p\left[\mathcal{S}_{y y}^{\alpha}\left(n_{0}\right) \mid \mathcal{H}_{1}\right](x)=\frac{1}{\theta_{1}^{\alpha}\left(n_{0}\right)} \cdot e^{-\frac{x}{\theta_{1}^{\alpha}\left(n_{0}\right)}},
$$

where, following the same reasoning as in the line case,

$$
\theta_{1}^{\alpha}\left(n_{0}\right)=\rho \mathcal{S}_{2}^{\alpha}\left(n_{0}\right)-\rho\left(1-\mu_{4}^{*}\right) \mathcal{S}_{1}^{\alpha}\left(n_{0}\right)+\sigma_{\omega}^{4}+2 \sigma_{\omega}^{2} .
$$

\section{Concluding remarks}

The knowledge of the aforementioned distributions, including the theoretical expression of the entailed parameters, allows us to derive in the next Section the theoretical performance analysis of the classical CAP-based detector.

Prior to that, we draw in Fig. 1 the theoretical mean and variance of the CAP under $\mathcal{H}_{1}$ in both the spectral line and noise cases. We compare it with some simulation results drawn in the same context with 10000 independent realizations. As expected, the theoretical and simulated curves perfectly match.

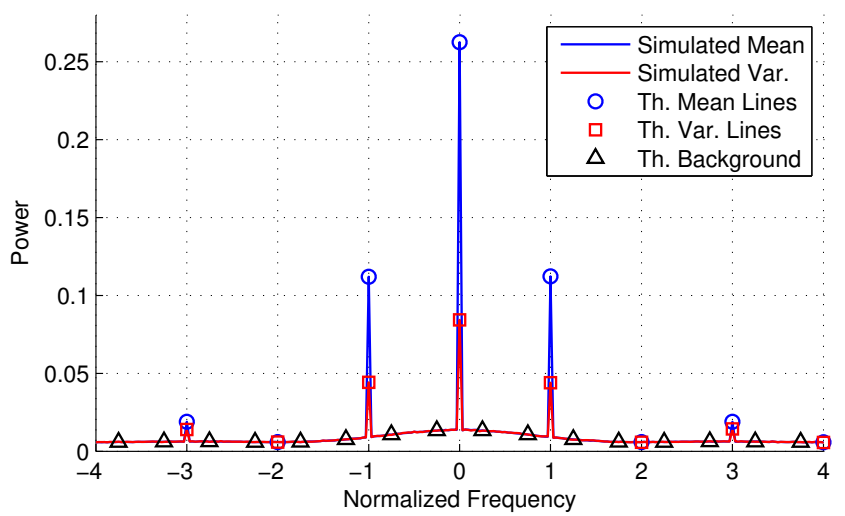

Fig. 1. Theoretical and simulated mean and variance of the cyclic autocorrelation power (CAP). The following parameters were used: $C=\mathrm{QPSK}$, $N=512, \mathrm{SNR}=0 \mathrm{~dB}, \rho=8, n_{0}=4$.

\section{THEORETICAL PERFORMANCE OF CAP DETECTION}

In this Section, we derive both the probabilities of detection $P_{d}=P\left[\mathcal{H}_{1} \mid \mathcal{H}_{1}\right]$ and false alarm $P_{f a}=P\left[\mathcal{H}_{1} \mid \mathcal{H}_{0}\right]$ for the classical CA-based spectrum sensing, i.e. a detector which relies on the power of the spectral line at null cyclic frequency.

\section{A. Theoretical $P_{d}$ and $P_{f a}$}

According to (16), the probability of false alarm for a given threshold $\xi$ is simply expressed as the complementary cumulative distribution function (CCDF) of the scaled Chisquare distribution with two degrees of freedom, yields:

$$
P_{f a}=e^{-\frac{\xi}{\sigma_{\omega}^{4}}}
$$

Conversely, it is possible to set threshold $\xi$ so that the detector respects some constraints on the probability of false alarm. We may straightforwardly get from (26):

$$
\xi=-\sigma_{\omega}^{4} \cdot \ln \left(P_{f a}\right)
$$

Furthermore, the probability of detection may be derived for all $n_{0} \in \llbracket 1 ;\lfloor\rho\rfloor \rrbracket$, according to the CCDF of the scaled non-central Chi-square distribution, as available in [12], as

$$
P_{d}=Q_{1}\left(\sqrt{\frac{2 \lambda_{1}^{0}\left(n_{0}\right)}{\theta_{1}^{0}\left(n_{0}\right)}}, \sqrt{\frac{2 \xi}{\theta_{1}^{0}\left(n_{0}\right)}}\right) .
$$

where $Q_{1}(\cdot, \cdot)$ stands for the generalized one-order Marcum Q function, and where we recall that $\lambda_{1}^{0}\left(n_{0}\right)$ and $\theta_{1}^{0}\left(n_{0}\right)$ are respectively given by (22) and (23).

\section{B. Adequacy between theory and simulation}

In this penultimate part, we confront the theoretical probabilities of detection $P_{d}$ (28) and false alarm $P_{f a}$ (26) with numerical simulations. Fig. 2 shows the theoretical and simulated $P_{d}$ and $P_{f a}$ as a function of the SNR and when the symbols are modulated via QPSK. The oversampling factor is set to $16, \tau_{0}$ is fixed to 0.125 (or equivalently $n_{0}=2$ ). For the simulations, 10000 independent trials were performed for each value of $\mathrm{N}$.

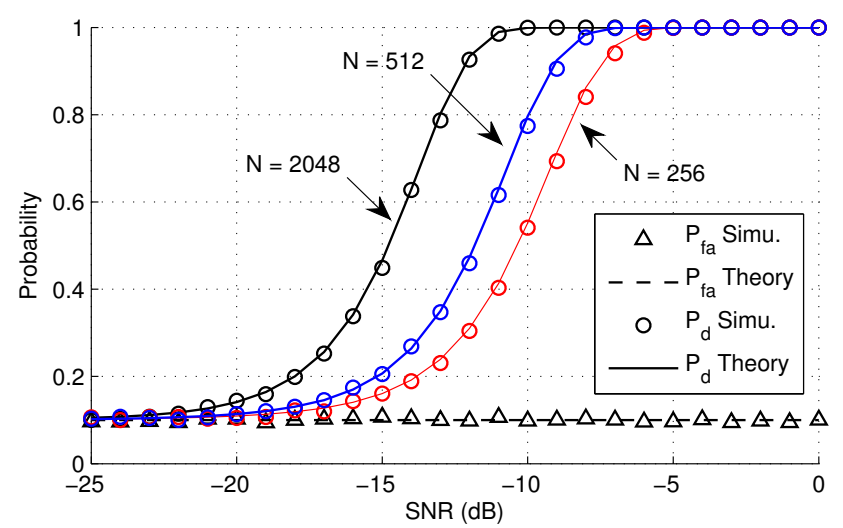

Fig. 2. Theoretical and simulated probabilities of detection $P_{d}$ and false alarm $P_{f a}$ for the classical CA-based detector, as a function of the SNR.

Finally, Fig. 3 shows the receiver operating characteristic (ROC) curves in the same context as the one of Fig. 1. As expected, the theoretical and simulated results match, even when the number of samples is fairly low. 


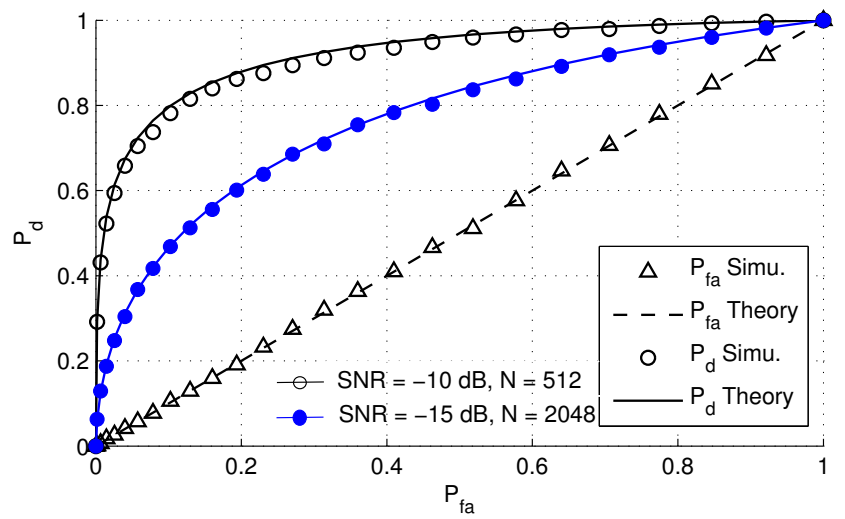

Fig. 3. Theoretical and simulated receiver operating characteristic (ROC) curves for the classical CA-based detector and for various parameters.

\section{CONCLUSION}

In this paper, we proposed a framework to derive the distributions of the cyclic autocorrelation power (CAP). We showed that this derivation allows to compute the theoretical probabilities of detection $P_{d}$ and false alarm $P_{f a}$ for the classical CA-based detector. These new results are particularly interesting in the field of spectrum sensing, where the performance must be closely studied to match some specific requirements. Further work shall be carried out based on this framework: for instance, theoretical results may be derived for other types of CA-based detectors or in the context of different assumptions on the received signal.

\section{APPENDIX}

For ease of notation, we denote in what follows

$$
h_{k}^{x} \triangleq h\left(t-x+k T_{s}+\tau / 2+\tau_{0} / 2\right) .
$$

From (4) and (5), we express the first term in (11) as

$$
\mathcal{S}_{a b, c d}^{\alpha}\left(\tau_{0}\right) \triangleq \mathcal{F}_{\tau}\left[\frac{1}{T_{s}} \int_{0}^{T_{s}}\left(\sum_{k} h_{k}^{0} h_{k}^{\tau_{0}}\right)\left(\sum_{k} h_{k}^{\tau} h_{k}^{\tau_{0}+\tau}\right) d t\right]
$$

where $\mathcal{F}_{\tau}[\cdot]$ is the Fourier Transform with respect to $\tau$.

According to the Poisson Summation Formula, we have:

$$
\begin{array}{r}
\sum_{k} h_{k}^{0} h_{k}^{\tau_{0}}=\frac{1}{T_{s}} \cdot \sum_{m \in f_{s} \mathbb{Z}} e^{i 2 \pi m t} H_{0, \tau_{0}}(m) \\
\sum_{k} h_{k}^{\tau} h_{k}^{\tau_{0}+\tau}=\frac{1}{T_{s}} \cdot \sum_{m \in f_{s} \mathbb{Z}} e^{i 2 \pi m t} H_{\tau, \tau_{0}+\tau}(m)
\end{array}
$$

where $H_{0, \tau_{0}}(f)=\mathcal{F}_{t}\left[h^{0} h^{\tau_{0}}\right]$ and $H_{\tau, \tau_{0}+\tau}(f)=\mathcal{F}_{t}\left[h^{\tau} h^{\tau_{0}+\tau}\right]$.

Remarking that $H_{\tau, \tau_{0}+\tau}(f)=e^{i 2 \pi \alpha \tau} H_{0, \tau_{0}}(f)$, we have:

$$
\begin{aligned}
\frac{1}{T_{s}} \int_{0}^{T_{s}} & \left(\sum_{k} h_{k}^{0} h_{k}^{\tau_{0}}\right)\left(\sum_{k} h_{k}^{\tau} h_{k}^{\tau_{0}+\tau}\right) d t \\
& =\frac{1}{T_{s}^{2}} \sum_{m \in f_{s} \mathbb{Z}} e^{-i 2 \pi m \tau} H_{0, \tau_{0}}(m) H_{0, \tau_{0}}(-m)
\end{aligned}
$$

We deduce that, for $\left.\tau_{0} \in\right]-T_{s} ; T_{s}[$ :

$$
\mathcal{S}_{a b, c d}^{\alpha}\left(\tau_{0}\right)=\frac{1}{T_{s}^{2}} \sum_{m \in f_{s} \mathbb{Z}} H_{0, \tau_{0}}^{2}(m) \int_{-\infty}^{\infty} e^{i 2 \pi \tau(\alpha-m)} d \tau
$$

Moreover, for $\left.\tau_{0} \in\right]-T_{s} ; T_{s}[$ :

$$
H_{0, \tau_{0}}(f)=\left(T_{s}-\tau_{0}\right) \cdot \operatorname{sinc}\left(\alpha\left(T_{s}-\tau_{0}\right)\right)
$$

Finally, for $\left.\tau_{0} \in\right]-T_{s} ; T_{s}[$ :

$$
\mathcal{S}_{a b, c d}^{\alpha}\left(\tau_{0}\right)=\left(\frac{T_{s}-\tau_{0}}{T_{s}}\right)^{2} \sum_{m \in f_{s} \mathbb{Z}} \operatorname{sinc}^{2}\left(m\left(T_{s}-\tau_{0}\right)\right) \delta(\alpha-m)
$$

where $\delta$ classically stands for the Dirac delta function.

\section{REFERENCES}

[1] T. Yucek and H. Arslan, "A Survey of Spectrum Sensing Algorithms for Cognitive Radio Applications," IEEE Communications Surveys Tutorials, vol. 11, no. 1, pp. 116-130, 2009.

[2] J. E. Mazo, "Jitter Comparison of Tones Generated by Squaring and by Fourth-power Circuits," The Bell System Technical Journal, vol. 57, no. 5, pp. 1489-1498, 1978.

[3] G. Gelli and L. Izzo, "Cyclostationarity-based Coherent Methods for Wideband-Signal Source Location," IEEE Transactions on Signal Processing, vol. 51, no. 10, pp. 2471-2482, 2003.

[4] B. Ramkumar, "Automatic Modulation Classification for Cognitive Radios using Cyclic Feature Detection," IEEE Circuits and Systems Magazine, vol. 9, no. 2, pp. 27-45, 2009.

[5] O. A. Dobre, M. Oner, S. Rajan, and R. Inkol, "Cyclostationarity-Based Robust Algorithms for QAM Signal Identification," IEEE Communications Letters, vol. 16, no. 1, pp. 12-15, 2012.

[6] C. W. Lim and M. B. Wakin, "Automatic Modulation Recognition for Spectrum Sensing using Nonuniform Compressive Samples," IEEE International Conference on Communications (ICC), pp. 3505-3510, 2012.

[7] J. K. Tugnait and G. Huang, "Cyclic Autocorrelation based Spectrum Sensing in Colored Gaussian Noise," IEEE Wireless Communications and Networking Conference (WCNC), pp. 731-736, 2012.

[8] W. A. Gardner and C. M. Spooner, "Signal Interception: Performance Advantages of Cyclic-Feature Detectors," IEEE Transactions on Communications, vol. 40, no. 1, pp. 149-159, 1992.

[9] G. Carter, C. Knapp, and A. Nuttall, "Estimation of the Magnitudesquared Coherence Function via Overlapped Fast Fourier Transform Processing," IEEE Transactions on Audio and Electroacoustics, vol. 21, no. 4, pp. 337-344, 1973.

[10] H. S. Chen, W. Gao, and D. G. Daut, "Spectrum Sensing using Cyclostationary Properties and Application to IEEE 802.22 WRAN,"' IEEE Global Telecommunications Conference (GLOBECOM), pp. 31333138, 2007.

[11] A. V. Dandawate and G. B. Giannakis, "Statistical Tests for Presence of Cyclostationarity," IEEE Transactions on Signal Processing, vol. 42 no. 9, pp. 2355-2369, 1994.

[12] S. M. Kay, "Fundamentals of Statistical Signal Processing, Volume 2: Detection Theory," Prentice-Hall, 1993.

[13] X. Chen, W. Xu, Z. He, and X. Tao, "Spectral Correlation-based Multiantenna Spectrum Sensing Technique," IEEE Wireless Communications and Networking Conference (WCNC), pp. 735-740, 2008.

[14] W.-J. Yue, B.-Y. Zheng, and Q.-M. Meng, "Cyclostationary Property based Spectrum Sensing algorithms for Primary Detection in Cognitive Radio Systems," Journal of Shanghai Jiaotong University (Science), vol. 14, no. 6, pp. 676-680, 2009.

[15] J. Reichert, "Automatic Classification of Communication Signals using Higher Order Statistics," IEEE International Conference on Acoustics, Speech, and Signal Processing (ICASSP), vol. 5, pp. 221-224 vol.5, 1992.

[16] J. Lunden, V. Koivunen, A. Huttunen, and H. V. Poor, "Spectrum Sensing in Cognitive Radios Based on Multiple Cyclic Frequencies," 2nd International Conference on Cognitive Radio Oriented Wireless Networks and Communications, pp. 37-43, 2007. 